\title{
REFINED HIGHER ORDER FINITE ELEMENT MODELS FOR THERMAL BUCKLING OF LAMINATED COMPOSITE AND SANDWICH PLATES
}

\author{
C. Sarath Babu \\ T. Kant \\ Department of Civil Engineering \\ Indian Institute of Technology Bombay \\ Mumbai, India
}

\begin{abstract}
Two refined higher order theories, one that neglects and the other that takes into account the effect of transverse normal deformation, are used to develop two discrete finite element models for the thermal buckling analysis of composite laminates and sandwiches. The two models, one with nine degrees of freedom per node and the other with eleven degrees of freedom, are based on a nine-node Lagrangian isoparametric element. The geometric stiffness matrices are developed by taking into consideration the effects of the higher order terms on the initial in-plane and transverse shear stresses. The accuracy of the present formulations is first evaluated by an alyzing sample problems for which analytical three-dimensional solutions exist in the literature. Numerical results are presented for the first time for sandwich plates, demonstrating the importance and accuracy of the higher order theory in comparison to first-order theory. Some new results are also given for sandwich plates with angle-ply composite face sheets, showing the effects of various boundary conditions and of variations in geometric and lamination parameters on critical temperature.
\end{abstract}

Fiber-reinforced composite laminates due to their high specific strength and stiffness are increasingly used in weight-sensitive applications such as aircraft and space vehicles. Most of these vehicles have to operate in hostile thermal environments; as a result, the structural components of these vehicles are subjected to thermal loads. In certain cases, the thermal load turns out to be the primary load, and the thermal stability of composite laminates is one of the factors governing their design.

Various laminated plate theories, depending upon the through-thickness displacement pattern considered, have been used to determine buckling loads of composite plates. The classical lamination plate theory (CLPT), which is based on Kirchhoff's hypothesis, overestimates the buckling load when applied to even moderately thick plates. This is particularly true for fiber-reinforced composite plates in which transverse shear moduli are small in comparison to the in-plane Young's moduli. In such cases, it becomes necessary to take into account shear

Received 2 December 1998; accepted 4 August 1999.

Address correspondence to Professor T. Kant, Department of Civil Engineering, Indian Institute of Technology Bombay, Powai, Mumbai-400076, India. E-mail: tkant@ civil.iitb.ernet.in 
deformation effects. Thus, various improved plate theories such as first-order shear deformation (FSDT) and higher order shear deformation (HSDT) theories have been developed to predict the behavior of plates with thickness shear deformation (see excellent survey articles by Tauchert [1], Noor and Burton [2], and Thornton [3] that include thermal buckling of shear deformable composite plates).

Probably the first analyses of thermal buckling of shear deformable laminated plates are included in the work of Tauchert [4]. He used FSDT to analyze simply supported plates of antisymmetric angle-ply construction subjected to a uniform temperature rise. Yang and Sheih $[5]$ employed the Galerkin method to investigate thermal buckling of initially stressed antisymmetric cross-ply plates. Chen et al. [6] considered both uniform and nonuniform temperature distributions using the finite element method. Noor and Burton [7] used predictor-corrector procedures for thermal buckling analysis. Prabhu and Dhanaraj [8] considered symmetrically laminated plates with different boundary conditions and used the finite element method in the analysis. The FSDT used in these studies predict buckling loads fairly accurately, provided proper values are selected for composite shear correction factors that are problem dependent. On the other hand, the higher order shear deformation theories that are based on the realistic through-the-thickness distribution of displacement components do not require shear correction coefficients and, in addition, incorporate the effects of transverse normal deformation.

The first work on thermal buckling of composite laminates using HSDT was the work of Chang [9]. The HSDT takes into account the thickness normal deformation effect, but the terms that represent the second-order variation of in-plane displacements through the thickness are not considered. Chang and Leu [10] used a theory that considers the transverse normal deformation and obtained analytical solutions for antisymmetric laminates using full stress/strain relations. The numerical results showed surprising discrepancies, almost independent of the slenderness, when compared with FSDT and Reddy's [11] third-order theory. The stress resultants corresponding to the transverse normal stress are not included in the thermally induced fundamental state of stress, which has lead to large differences. Later Rohwer [12] corrected this by using a reduced stress/strain relationship. Shu and Sun [13] obtained a classical analytical solution for simply supported symmetric cross-ply laminates using a higher order formulation that accounts for the parabolic variation of transverse shear strains and shear stress continuity across each layer interface. All of the investigators in these studies, based on HSDT, obtained classical analytical solutions except in [9] where the finite element method was adopted.

Compared with the literature on high-temperature multilayered composite plates, very little is reported on thermal buckling analyses of sandwich plates with composite face sheets. The exception is the work of Noor et al. [14], who presented three-dimensional solutions for the buckling of simply supported sandwiches subjected to thermal and mechanical loads. The effect of temperature on the mechanical buckling load was investigated by Ko and Jackson [15] using the Rayleigh-Ritz minimum energy method with classical sandwich plate theory. Various computational models used for the analysis of sandwiches were summarized in the recently published review article of Noor et al. [16], which includes thermal buckling. Most 
of the investigations for predicting the response characteristics of sandwich plates use the classical sandwich plate theory in which the thickness shear deformation of face sheets and the membrane and bending stiffnesses of core are neglected. Increasing core stiffness can significantly contribute to the overall membrane and bending stiffness, while using composite materials in relatively thick face layers should be followed by incorporating transverse shear effects in the face layers. These effects can be taken into account by HSDTs. Although several higher order theories have been proposed for the thermal buckling analysis of composite laminates, no serious effort has been made thus far to apply these theories to a conventional sandwich plate that has a central soft core between two stiff face layers. Here an attempt is made to fill the void.

In the present article, thermal buckling analyses of multilayered composite plates and sandwiches using two discrete finite element models is presented. The finite element models are based on two refined higher order theories [17-22], one that neglects the effect of transverse normal deformation and one that considers that effect. Results obtained by these theories are first compared with three-dimensional solutions available in the literature, and the effect of the inclusion of transverse normal deformation on the buckling load is discussed. Additional numerical results are presented for sandwich plates that show the effects of variations in the geometric and lamination parameters on their thermal buckling response under different boundary conditions.

\section{FORMULATION}

\section{Displacement Models}

Two refined higher order theories, one that neglects the effect of transverse normal deformation and one that considers this effect, that are considered in this article are based on the assumption of the displacement fields in the following forms (Figure 1):

i. Higher order shear deformation theory (HOST9), 9 dof/node

$$
\begin{aligned}
& u(x, y, z)=u_{0}(x, y)+z \theta_{y}(x, y)+z^{2} u_{0}^{*}(x, y)+z^{3} \theta_{y}^{*}(x, y) \\
& v(x, y, z)=v_{0}(x, y)-z \theta_{x}(x, y)+z^{2} v_{0}^{*}(x, y)-z^{3} \theta_{x}^{*}(x, y) \\
& w(x, y, z)=w_{0}(x, y)
\end{aligned}
$$

ii. Higher order shear deformation theory (HOST11), 11 dof/node

$$
\begin{aligned}
& u(x, y, z)=u_{0}(x, y)+z \theta_{y}(x, y)+z^{2} u_{0}^{*}(x, y)+z^{3} \theta_{y}^{*}(x, y) \\
& v(x, y, z)=v_{0}(x, y)-z \theta_{x}(x, y)+z^{2} v_{0}^{*}(x, y)-z^{3} \theta_{x}^{*}(x, y) \\
& w(x, y, z)=w_{0}(x, y)+z \theta_{z}(x, y)+z^{2} w_{0}^{*}(x, y)
\end{aligned}
$$



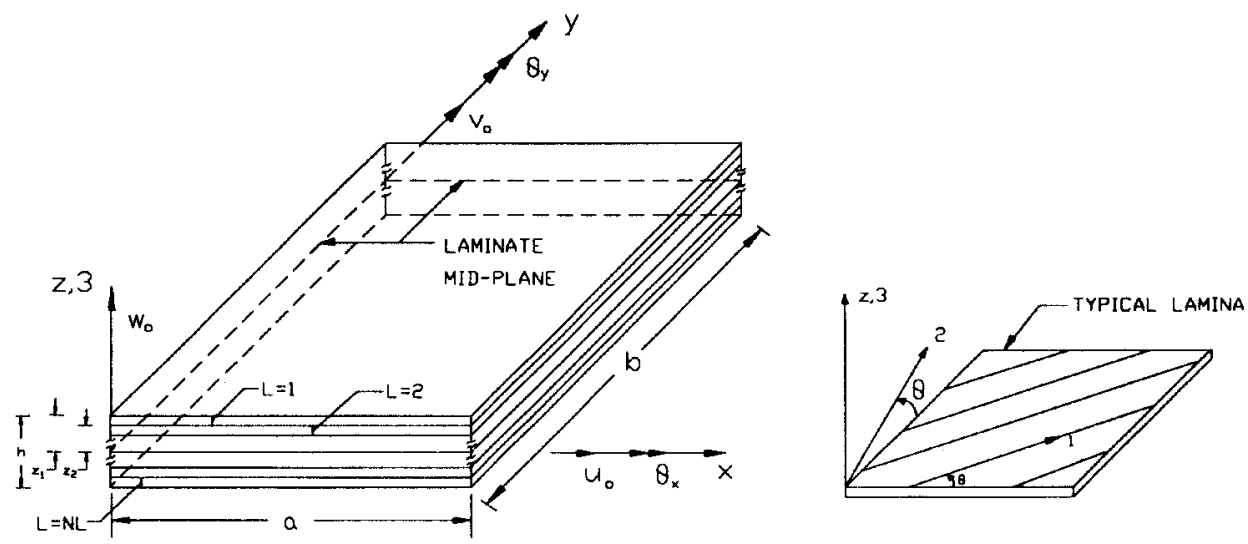

$(\mathrm{x}, \mathrm{y}, \mathrm{z})$ - LAMINATE REFERENCE AXES

$(1,2,3)$ - LAMINA REFERENCE AXES

Figure 1. Laminate geometry with positive set of lamina/laminate reference axes, displacement components, and fiber orientation.

where $u, v$, and $w$ define the displacements of any generic point $(x, y, z)$ in the plate space; $u_{0}, v_{0}$, and $w_{0}$ denote the displacements of a point $(x, y)$ on the middle plane; and $\theta_{x}$ and $\theta_{y}$ are the rotations of the normal to middle-plane about the $x$ and $y$-axes, respectively. The parameters $u_{0}^{*}, v_{0}^{*}, w_{0}^{*}, \theta_{x}^{*}, \theta_{y}^{*}$, and $\theta_{z}$ are higher order terms in the Taylor's series expansion and are defined at midsurface. The continuum displacement vector at the midplane can thus be defined as

$$
\begin{gathered}
\mathbf{u}=\left\{u_{0}, v_{0}, w_{0}, \theta_{x}, \theta_{y}, u_{0}^{*}, v_{0}^{*}, \theta_{x}^{*}, \theta_{y}^{*}\right\}^{T} \text { for HOST9 } \\
\mathbf{u}=\left\{u_{0}, v_{0}, w_{0}, \theta_{x}, \theta_{y}, \theta_{z}, u_{0}^{*}, v_{0}^{*}, w_{0}^{*}, \theta_{x}^{*}, \theta_{y}^{*}\right\}^{T} \text { for HOST11 }
\end{gathered}
$$

It should be noted that the additional degrees of freedom such as $\theta_{z}$ and $w_{0}^{*}$ in HOST11 account for the transverse normal deformation effect while HOST9 does not.

\section{Stress-Strain Relationship}

The stress-strain relations $[23]$ for the Lth lamina in the laminate coordinates $(x, y, z)$ are written as

$$
\left\{\begin{array}{c}
\sigma_{x} \\
\sigma_{y} \\
\sigma_{z} \\
\tau_{x y} \\
\tau_{y z} \\
\tau_{x z}
\end{array}\right\}=\left[\begin{array}{cccccc}
Q_{11} & Q_{12} & Q_{13} & Q_{14} & 0 & 0 \\
Q_{12} & Q_{22} & Q_{23} & Q_{24} & 0 & 0 \\
Q_{13} & Q_{23} & Q_{33} & Q_{34} & 0 & 0 \\
Q_{14} & Q_{24} & Q_{34} & Q_{44} & 0 & 0 \\
0 & 0 & 0 & 0 & Q_{55} & Q_{56} \\
0 & 0 & 0 & 0 & Q_{56} & Q_{66}
\end{array}\right]\left\{\begin{array}{c}
\varepsilon_{x}-\alpha_{x} \Delta T \\
\varepsilon_{y}-\alpha_{y} \Delta T \\
\varepsilon_{z}-\alpha_{z} \Delta T \\
\gamma_{x y}-\alpha_{x y} \Delta T \\
\gamma_{y z} \\
\gamma_{x z}
\end{array}\right\}
$$


or in short form

$$
\sigma=\mathbf{Q}\left(\epsilon-\epsilon_{\mathbf{t}}\right)
$$

in which

$$
\begin{aligned}
\sigma & =\left\{\begin{array}{llllll}
\sigma_{x} & \sigma_{y} & \sigma_{z} & \tau_{x y} & \tau_{y z} & \tau_{x z}
\end{array}\right\}^{\mathrm{T}} \\
\boldsymbol{\epsilon} & =\left\{\begin{array}{lllllll}
\varepsilon_{x} & \varepsilon_{y} & \varepsilon_{z} & \gamma_{x y} & \gamma_{y z} & \gamma_{x z}
\end{array}\right\}^{\mathrm{T}} \\
\boldsymbol{\epsilon}_{\mathbf{t}} & =\left\{\begin{array}{lllllll}
\alpha_{x} & \alpha_{y} & \alpha_{z} & \alpha_{x y} & 0 & 0
\end{array}\right\}^{\mathrm{T}} \Delta T
\end{aligned}
$$

are, respectively, the stress, the total strain, and the thermal strain vectors. $Q_{i j}$ 's are the transformed stiffness coefficients. The transformation of the stresses / strains between the lamina and laminate coordinate systems follows the usual stress tensor transformation rule. $\Delta T$ is the temperature rise. $\alpha_{x}, \alpha_{y}, \alpha_{z}$, and $\alpha_{x y}$ are defined as

$$
\begin{array}{ll}
\alpha_{x}=\alpha_{1} \cos ^{2} \theta+\alpha_{2} \sin ^{2} \theta & \alpha_{y}=\alpha_{1} \sin ^{2} \theta+\alpha_{2} \cos ^{2} \theta \\
\alpha_{z}=\alpha_{3} & \alpha_{x y}=2\left(\alpha_{1}-\alpha_{2}\right) \sin \theta \cos \theta
\end{array}
$$

where $\alpha_{1}, \alpha_{2}$, and $\alpha_{3}$ are the thermal expansion coefficients in the principal directions of lamina and $\theta$ is the angle between the lamina and laminate axes. If the transverse normal stress and strain are neglected, the stress-strain relations for the $L$ th lamina in the laminate coordinates $(x, y, z)$ can be expressed as

$$
\left\{\begin{array}{c}
\sigma_{x} \\
\sigma_{y} \\
\tau_{x y} \\
\tau_{y z} \\
\tau_{x z}
\end{array}\right\}=\left[\begin{array}{ccccc}
Q_{11}^{\prime} & Q_{12}^{\prime} & Q_{13}^{\prime} & 0 & 0 \\
Q_{12}^{\prime} & Q_{22}^{\prime} & Q_{23}^{\prime} & 0 & 0 \\
Q_{13}^{\prime} & Q_{23}^{\prime} & Q_{33}^{\prime} & 0 & 0 \\
0 & 0 & 0 & Q_{44}^{\prime} & Q_{45}^{\prime} \\
0 & 0 & 0 & Q_{45}^{\prime} & Q_{55}^{\prime}
\end{array}\right]\left\{\begin{array}{c}
\varepsilon_{x}-\alpha_{x} \Delta T \\
\varepsilon_{y}-\alpha_{y} \Delta T \\
\gamma_{x y}-\alpha_{x y} \Delta T \\
\gamma_{y z} \\
\gamma_{x z}
\end{array}\right\}
$$

in which $Q_{i j}^{\prime}$ s are the plane stress-reduced stiffness coefficients. This constitutive relation, which does not take into account the transverse normal strain and thermal expansion effect in the thickness direction, is used along with HOST9, while the constitutive relation (5), which takes account of these effects, is used with HOST11. It will be noted that the formulation given in the rest of the article is based on HOST9 and Eq. (9).

\section{Strain-Displacement Relationship}

Substituting Eq. (1) into the Green's strain tensor [24], with $\varepsilon_{z}=0$, the generalized strain vector components are obtained as

$$
\begin{aligned}
& \varepsilon_{x}=\varepsilon_{x}^{0}+z \chi_{x}^{0}+z^{2} \mathcal{E}_{x}^{*}+z^{3} \chi_{x}^{*}+z^{4} \mathcal{E}_{x}^{* *}+z^{5} \chi_{x}^{* *}+z^{6} \varepsilon_{x}^{* * *} \\
& \varepsilon_{y}=\varepsilon_{y}^{0}+z \chi_{y}^{0}+z^{2} \varepsilon_{y}^{*}+z^{3} \chi_{y}^{*}+z^{4} \mathcal{E}_{y}^{* *}+z^{5} \chi_{y}^{* *}+z^{6} \varepsilon_{y}^{* * *}
\end{aligned}
$$




$$
\begin{aligned}
& \gamma_{x y}=\varepsilon_{x y}^{0}+z \chi_{x y}^{0}+z^{2} \varepsilon_{x y}^{*}+z^{3} \chi_{x y}^{*}+z^{4} \mathcal{E}_{x y}^{* *}+z^{5} \chi_{x y}^{* *}+z^{6} \varepsilon_{x y}^{* * *} \\
& \gamma_{y z}=\phi_{y}^{0}+z \psi_{y}^{0}+z^{2} \phi_{y}^{*}+z^{3} \psi_{y}^{*}+z^{4} \phi_{y}^{* *}+z^{5} \psi_{y}^{* *} \\
& \gamma_{x z}=\phi_{x}^{0}+z \psi_{x}^{0}+z^{2} \phi_{x}^{*}+z^{3} \psi_{x}^{*}+z^{4} \phi_{x}^{* *}+z^{5} \psi_{x}^{* *}
\end{aligned}
$$

Note that the five generalized strain components are expressed in terms of 33 strain components denoted by the vector, $\overline{\boldsymbol{\epsilon}}$ (Appendix). Each of the components of $\boldsymbol{\epsilon}$ has both linear as well as nonlinear parts that can be expressed in terms of midplane displacement components.

The potential energy, $\Pi$, of the plate can be expressed as

$$
\Pi=\frac{1}{2} \int_{v}\left\{\boldsymbol{\epsilon}-\boldsymbol{\epsilon}_{\mathbf{t}}\right\}^{\mathrm{T}} \sigma d v-\int_{v} \mathbf{u}^{\mathrm{T}} \mathbf{p} d v
$$

where $\mathbf{p}$ is the vector of in-plane mechanical loads.

Substituting for $\epsilon$ from Eq. (10) and $\sigma$ from Eq. (9) in Eq. (11) and performing an explicit integration through the laminate thickness give the expression

$$
\Pi=\frac{1}{2} \int_{A} \bar{\varepsilon}^{\mathrm{T}} \bar{\sigma} d A-\frac{1}{2} \int_{A} \bar{\varepsilon}^{\mathrm{T}} \bar{\sigma}_{t} d A-\int_{A} \mathbf{u}^{\mathrm{T}} \mathbf{p} d A+f\left(Q_{i j}, \alpha_{i}\right)
$$

in which $f$ is a function of $Q_{i j}$ and $\alpha_{i}$ and the new vectors in concise form (see the Appendix for details) are expressed as

$$
\bar{\sigma}=\left\{\begin{array}{llllll}
\mathbf{N}^{\mathrm{T}} & \mathbf{M}^{\mathrm{T}} & \mathbf{Q}^{\mathrm{T}}
\end{array}\right\}^{\mathrm{T}} \quad \bar{\sigma}_{\mathbf{t}}=\left\{\begin{array}{llll}
\mathbf{N}_{t}^{\mathrm{T}} & \mathbf{M}_{t}^{\mathrm{T}} & \mathbf{O}_{t}^{\mathrm{T}}
\end{array}\right\}^{\mathrm{T}} \quad \overline{\boldsymbol{\epsilon}}=\left\{\begin{array}{lll}
\boldsymbol{\epsilon}_{0}^{\mathrm{T}} & \chi^{\mathrm{T}} & \boldsymbol{\psi}^{\mathrm{T}}
\end{array}\right\}^{\mathrm{T}}
$$

The stress resultants in Eq. (13) are defined as

$$
\begin{aligned}
& {\left[\begin{array}{ccc}
N_{x}^{0} & N_{y}^{0} & N_{x y}^{0} \\
N_{x}^{*} & N_{y}^{*} & N_{x y}^{*} \\
N_{x}^{* *} & N_{y}^{* *} & N_{x y}^{* *} \\
N_{x}^{* * *} & N_{y}^{* * *} & N_{x y}^{* * *}
\end{array}\right]=\sum_{L=1}^{N L} \int_{Z_{L}}^{Z_{L+1}}\left[\begin{array}{l}
1 \\
z^{2} \\
z^{4} \\
z^{6}
\end{array}\right]\left[\begin{array}{lll}
\sigma_{x} & \sigma_{y} & \tau_{x y}
\end{array}\right] d z} \\
& {\left[\begin{array}{ccc}
M_{x}^{0} & M_{y}^{0} & M_{x y}^{0} \\
M_{x}^{*} & M_{y}^{*} & M_{x y}^{*} \\
M_{x}^{* *} & M_{y}^{* *} & M_{x y}^{* *}
\end{array}\right]=\sum_{L=1}^{N L} \int_{Z_{L}}^{Z_{L+1}}\left[\begin{array}{l}
z \\
z^{3} \\
z^{5}
\end{array}\right]\left[\begin{array}{lll}
\sigma_{x} & \sigma_{y} & \tau_{x y}
\end{array}\right] d z}
\end{aligned}
$$




$$
\left[\begin{array}{cc}
Q_{x}^{0} & Q_{y}^{0} \\
S_{x}^{0} & S_{y}^{0} \\
Q_{x}^{*} & Q_{y}^{*} \\
S_{x}^{*} & S_{y}^{*} \\
Q_{x}^{* *} & Q_{y}^{* *} \\
S_{x}^{* *} & S_{y}^{* *}
\end{array}\right]=\sum_{L=1}^{N L} \int_{Z_{L}}^{Z_{L+1}}\left[\begin{array}{c}
1 \\
z \\
z^{2} \\
z^{3} \\
z^{4} \\
z^{5}
\end{array}\right]\left[\begin{array}{ll}
\tau_{x z} & \tau_{y z}
\end{array}\right] d z
$$

After integration, these stress resultants are written in matrix form as

$$
\bar{\sigma}=\mathbf{D} \bar{\epsilon}-\bar{\sigma}_{\mathbf{t}}
$$

where

$$
\mathbf{D}=\left[\begin{array}{ccc}
\mathbf{D}_{M} & \mathbf{D}_{C} & 0 \\
\mathbf{D}_{C}^{\mathrm{T}} & \mathbf{D}_{B} & 0 \\
0 & 0 & \mathbf{D}_{S}
\end{array}\right]
$$

in which $\mathbf{D}_{M}, \mathbf{D}_{B}, \mathbf{D}_{C}$, and $\mathbf{D}_{S}$ are the membrane, flexural, membrane-flexural coupling, and shear rigidity matrices, respectively. The elements of these rigidity matrices are given in the appendix. The vector, $\sigma_{t}$, in Eq. (13) represents the thermal stress resultants (see the Appendix).

\section{FINITE ELEMENT FORMULATION}

Let the region of the plate be divided into a finite number of quadrilateral elements. The continuum displacement vector within the element is discretized such that

$$
\mathbf{u}=\sum_{i=1}^{N N} N_{i} \mathbf{u}_{i}
$$

where $N_{i}(\xi, \eta)$ is the shape function of node $i, N N$ is the number of nodes in an element, and $\mathbf{u}_{i}$ is the generalized displacement vector corresponding to the $i$ th node of an element. This relation is expressed in matrix form as

$$
\mathbf{u}=\overline{\mathbf{N}} \mathbf{d}
$$

where $\overline{\mathbf{N}}$ is the element shape function matrix and $\mathbf{d}$ is the element nodal displacement vector. The components of the strain vector, $\mathbf{\epsilon}$, can be expressed in 
terms of nodal displacement vector, $\mathbf{d}$, as

$$
\bar{\epsilon}=\left[\mathbf{B}_{0}+\frac{1}{2} \mathbf{B}_{L}\right] \mathbf{d}
$$

where $\mathbf{B}_{0}$ and $\mathbf{B}_{L}$ are linear and nonlinear strain-displacement matrices. Substituting for $\overline{\boldsymbol{\epsilon}}$ from Eqs. (21) in (12) and then minimizing potential energy give

$$
\sum_{i=1}^{n}\left[\mathbf{K}_{0}^{\mathrm{e}} \mathbf{d}+\mathbf{K}_{\mathrm{g}}^{\mathrm{e}} \mathbf{d}-\mathbf{R}^{\mathrm{e}}\right]=0
$$

where $n$ is the number of elements, $\mathbf{K}_{0}^{\mathrm{e}}$ and $\mathbf{K}_{\mathrm{g}}^{\mathrm{e}}$ are the linear and geometric element stiffness matrices, and $\mathbf{R}^{\mathrm{e}}$ is the element load vector. These matrices are constructed using the standard procedure $[24]$ as

$$
\begin{gathered}
\mathbf{K}_{0}^{\mathrm{e}}=\int_{A} \mathbf{B}_{0}^{\mathrm{T}} \mathbf{D} \mathbf{B}_{0} d A \\
\mathbf{K}_{\mathrm{g}}^{\mathrm{e}} \mathbf{d}=\int_{A} \mathbf{B}_{L}^{\mathrm{T}} \mathbf{S} d A \\
\mathbf{R}^{\mathrm{e}}=\int_{A} \mathbf{B}_{0}^{\mathrm{T}} \overline{\boldsymbol{\sigma}}_{\mathbf{t}} d A+\int_{A} \mathbf{N}^{\mathrm{T}} \mathbf{p} d A
\end{gathered}
$$

where $\mathbf{S}$ is the stress resultant matrix.

Following the usual procedure for assembling element stiffness matrices, the equilibrium and stability conditions are expressed as

$$
\begin{gathered}
\mathbf{K}_{0} \overline{\mathbf{d}}=\mathbf{R} \\
{\left[\mathbf{K}_{0}+\lambda \mathbf{K}_{\mathrm{g}}\right] \delta \overline{\mathbf{d}}=0}
\end{gathered}
$$

where $\mathbf{K}_{0}, \mathbf{K}_{\mathrm{g}}$, and $\mathbf{R}$ are, respectively, the global stiffness matrix, the global ge ometric stiffness matrix, and the global load vectors and $\overline{\mathbf{d}}$ is the nodal displacement vector of the plate.

\section{Thermal Buckling Analysis}

Calculating the critical temperature of buckling due to thermal load is a two-stage process. For a specified rise $(\Delta T)$ in temperature the thermal loads are computed (25) and a linear static analysis (26) is carried out to determine the thermal stress resultants. These stress resultants are then used to compute the geometric stiffness matrix, which is subsequently used in Eq. (27), to determine the least eigenvalue, $\lambda$, and the associated mode shape, $\delta \overline{\mathbf{d}}$. The critical temperature, $T_{\mathrm{cr}}$, of the plate is calculated using

$$
T_{c r}=\lambda \Delta T
$$




\section{NUMERICAL RESULTS AND DISCUSSION}

Computer programs are developed based on the foregoing finite element models to solve a number of examples on thermal buckling of composite and sandwich plates. The programs can handle panels subjected to nonuniform temperature rise over the surface and through the thickness. However, in all the examples solved here, the temperature rise is assumed to be uniform. A nine-noded Lagrangian element is used. The selective integration scheme-namely, the $3 \times 3$ Gauss-Legendre for membrane, flexure, and membrane-flexure and $2 \times 2$ for shear contributions of energy - is used for plates that have a width-to-thickness ratio greater than 20 ; in all other cases the full integration scheme $(3 \times 3)$ is used. A full plate model of 36 $(6 \times 6 \mathrm{mesh})$ elements is used in the analyses. This scheme is arrived at on the basis of a convergence study in which the critical temperature converges monotonically from a higher value. The details of the convergence study is not presented for the sake of brevity. The boundary conditions used in the analyses are specified in Table 1. A parallel formulation based on Reissner-Mindlin first-order shear theory (FOST) is also developed in order to compare its results with those of higher order theories. A shear correction factor of $5 / 6$ is used with this theory.

Three numerical examples are presented here. The accuracy of the present higher order finite element formulations is evaluated in Examples 1 and 2 for composite laminates and sandwich panels, respectively. It is observed that except for the three-dimensional elasticity solutions, no other numerical results are reported in the literature on thermal buckling of sandwich panels with composite face sheets. In view of this, parametric studies on sandwiches with angle-ply composite face sheets are presented in Example 3.

Table 1 Details of boundary conditions for laminated plates and sandwiches

\begin{tabular}{|c|c|c|c|c|c|}
\hline \multirow[b]{2}{*}{ Boundary } & \multirow[b]{2}{*}{ Model } & \multicolumn{2}{|c|}{ Simply supported } & \multicolumn{2}{|c|}{ Fixed } \\
\hline & & $\mathrm{SS} 1$ & $\mathrm{SS} 2$ & $\mathrm{C} 1$ & $\mathrm{C} 2$ \\
\hline \multirow[t]{2}{*}{$x=0, a$} & HOST9 & $\begin{array}{l}v_{0}=w_{0}=0 \\
\theta_{x}=v_{0}^{*}=0 \\
\theta_{x}^{*}=0\end{array}$ & $\begin{array}{l}u_{0}=w_{0}=0 \\
\theta_{x}=u_{0}^{*}=0 \\
\theta_{x}^{*}=0\end{array}$ & $\begin{array}{l}v_{0}=w_{0}=0 \\
\theta_{x}=\theta_{y}=0 \\
v_{0}^{*}=\theta_{x}^{*}=0 \\
\theta_{y}^{*}=0\end{array}$ & $\begin{array}{l}u_{0}=w_{0}=0 \\
\theta_{x}=\theta_{y}=0 \\
u_{0}^{*}=\theta_{x}^{*}=0 \\
\theta_{y}^{*}=0\end{array}$ \\
\hline & HOST11 & $\begin{array}{c}v_{0}=w_{0}=0 \\
\theta_{x}=v_{0}^{*}=0 \\
w_{0}^{*}=\theta_{x}^{*}=0\end{array}$ & $\begin{array}{c}u_{0}=w_{0}=0 \\
\theta_{x}=u_{0}^{*}=0 \\
w_{0}^{*}=\theta_{x}^{*}=0\end{array}$ & $\begin{array}{l}v_{0}=w_{0}=0 \\
\theta_{x}=\theta_{y}=0 \\
v_{0}^{*}=w_{0}^{*}=0 \\
\theta_{x}^{*}=\theta_{y}^{*}=0\end{array}$ & $\begin{array}{l}u_{0}=w_{0}=0 \\
\theta_{x}=\theta_{y}=0 \\
u_{0}^{*}=w_{0}^{*}=0 \\
\theta_{x}^{*}=\theta_{y}^{*}=0\end{array}$ \\
\hline \multirow[t]{2}{*}{$y=0, b$} & HOST9 & $\begin{array}{l}u_{0}=w_{0}=0 \\
\theta_{y}=u_{0}^{*}=0 \\
\theta_{y}^{*}=0\end{array}$ & $\begin{array}{l}v_{0}=w_{0}=0 \\
\theta_{y}=v_{0}^{*}=0 \\
\theta_{y}^{*}=0\end{array}$ & $\begin{array}{l}u_{0}=w_{0}=0 \\
\theta_{x}=\theta_{y}=0 \\
u_{0}^{*}=\theta_{x}^{*}=0 \\
\theta_{y}^{*}=0\end{array}$ & $\begin{array}{l}v_{0}=w_{0}=0 \\
\theta_{x}=\theta_{y}=0 \\
v_{0}^{*}=\theta_{x}^{*}=0 \\
\theta_{y}^{*}=0\end{array}$ \\
\hline & HOST11 & $\begin{array}{c}u_{0}=w_{0}=0 \\
\theta_{y}=u_{0}^{*}=0 \\
w_{0}^{*}=\theta_{y}^{*}=0\end{array}$ & $\begin{array}{l}v_{0}=w_{0}=0 \\
\theta_{y}=v_{0}^{*}=0 \\
w_{0}^{*}=\theta_{y}^{*}=0\end{array}$ & $\begin{array}{c}u_{0}=w_{0}=0 \\
\theta_{x}=\theta_{y}=0 \\
u_{0}^{*}=w_{0}^{*}=0 \\
\theta_{x}^{*}=\theta_{y}^{*}=0\end{array}$ & $\begin{array}{l}v_{0}=w_{0}=0 \\
\theta_{x}=\theta_{y}=0 \\
v_{0}^{*}=w_{0}^{*}=0 \\
\theta_{x}^{*}=\theta_{y}^{*}=0\end{array}$ \\
\hline
\end{tabular}


Example 1: Simply supported, square, angle-ply composite plates for which analytical three-dimensional solutions $[25]$ for thermal buckling exist are considered here. The plates consist of 10 layers of equal thickness arranged antisymmetrically with respect to the middle plane. It is appropriate to mention here that true bifurcation type buckling cannot physically occur for antisymmetric laminates due to the presence of bending-extension coupling. However, the coupling effect is small for the 10-layered laminate considered here and this particular problem is solved for the purpose of comparison. The material characteristics of individual layers of the laminate are

$$
\begin{array}{lll}
E_{1} / E_{2}=15, E_{3}=E_{2} & G_{12} / E_{2}=G_{13} / E_{2}=0.50 & G_{23} / E_{2}=0.3356 \\
v_{12}=v_{13}=0.3, v_{23}=0.49 & \alpha_{1} / \alpha_{0}=0.015 & \alpha_{2} / \alpha_{0}=\alpha_{3} / \alpha_{0}=1.0
\end{array}
$$

where $\alpha_{0}$ is a normalization factor for the coefficients of thermal expansion. Thermal buckling stability parameters, $\lambda_{\mathrm{T}}=\alpha_{0} \mathrm{~T}_{\mathrm{Cr}}$ of the plates are determined using the present higher order finite element formulations and first-order theory. The results are compared with the three-dimensional elasticity solutions in Table 2 . The simply supported boundary conditions considered are of type SS2 (See Table 1). Both thin $(a / h=100)$ as well as thick $(a / h=5$ and 10) plates are considered with different fiber orientation angles.

The results of the present higher order models and FOST show very good agreement with the three-dimensional elasticity solution both for thin and thick laminates. In the case of thin plates, the results obtained with all the models are identical to the elasticity solution; and in thick plates $(a / h \leq 10)$, the differences are less than $2 \%$. It should be noted that though the differences in the values of the present models are negligible, the results obtained with the HOST9 model are closer to three-dimensional elasticity results. The results indicate that the inclusion

Table 2 Stability parameter for critical temperature, $\lambda_{\mathrm{T}}=\alpha_{0} T_{\mathrm{cr}}$, of simply supported antisymmetric angle-ply laminates $(N L=10$ and $a / b=1)$

\begin{tabular}{ccllll}
\hline$a / h$ & $\theta$ & $3 \mathrm{D}[20]$ & HOST9 & HOST11 & FOST \\
\hline 100 & 0 & $0.7463 \mathrm{E}-03$ & $0.7470 \mathrm{E}-03$ & $0.7471 \mathrm{E}-03$ & $0.7470 \mathrm{E}-03$ \\
& 15 & $0.1115 \mathrm{E}-02$ & $0.1116 \mathrm{E}-02$ & $0.1116 \mathrm{E}-02$ & $0.1116 \mathrm{E}-02$ \\
& 30 & $0.1502 \mathrm{E}-02$ & $0.1502 \mathrm{E}-02$ & $0.1502 \mathrm{E}-02$ & $0.1502 \mathrm{E}-02$ \\
& 45 & $0.1674 \mathrm{E}-02$ & $0.1675 \mathrm{E}-02$ & $0.1675 \mathrm{E}-02$ & $0.1675 \mathrm{E}-02$ \\
\hline \multirow{2}{*}{10} & 0 & $0.5782 \mathrm{E}-01$ & $0.5778 \mathrm{E}-01$ & $0.5817 \mathrm{E}-01$ & $0.5771 \mathrm{E}-01$ \\
& 15 & $0.7904 \mathrm{E}-01$ & $0.7920 \mathrm{E}-01$ & $0.7972 \mathrm{E}-01$ & $0.7941 \mathrm{E}-01$ \\
& 30 & $0.1100 \mathrm{E}-00$ & $0.1108 \mathrm{E}-00$ & $0.111 \mathrm{E}-00$ & $0.1114 \mathrm{E}-00$ \\
& 45 & $0.1194 \mathrm{E}-00$ & $0.1208 \mathrm{E}-00$ & $0.1211 \mathrm{E}-00$ & $0.1215 \mathrm{E}-00$ \\
\hline 5 & 0 & $0.1436 \mathrm{E}-00$ & $0.1417 \mathrm{E}-00$ & $0.1441 \mathrm{E}-00$ & $0.1409 \mathrm{E}-00$ \\
& 15 & $0.1753 \mathrm{E}-00$ & $0.1746 \mathrm{E}-00$ & $0.1773 \mathrm{E}-00$ & $0.1747 \mathrm{E}-00$ \\
& 30 & $0.2377 \mathrm{E}-00$ & $0.2421 \mathrm{E}-00$ & $0.2449 \mathrm{E}-00$ & $0.2424 \mathrm{E}-00$ \\
& 45 & xxxxxxxxxx & $0.2651 \mathrm{E}-00$ & $0.2667 \mathrm{E}-00$ & $0.2675 \mathrm{E}-00$ \\
\hline
\end{tabular}


of higher order degrees of freedom in the thickness direction in HOST11 does not improve the accuracy of the results.

Example 2: Simply supported (SS2) structural sandwiches for which three-dimensional elasticity solutions $[14,16]$ for thermal buckling exist are considered in the present example. The sandwiches are square in planform and made up of cross-ply composite face sheets with $N L=10$ and a honeycomb core. The fiber orientation and thicknesses of face sheets are such that the entire sandwich is symmetric with respect to the middle plane. The material characteristics of the face sheets are

$$
\begin{array}{lll}
E_{1} / E_{2}=19 & G_{12} / E_{2}=0.52 & G_{23} / E_{2}=0.338 \\
v_{12}=0.32 & v_{13}=v_{23}=0.49 & \alpha_{1} / \alpha_{0}=0.001, \alpha_{2} / \alpha_{0}=\alpha_{3} / \alpha_{0}=1.0
\end{array}
$$

and the material characteristics of the core are

$$
\begin{array}{lll}
E_{1} / E_{2}^{\mathrm{f}}=3.2 \times 0^{-5} & E_{2} / E_{2}^{\mathrm{f}}=2.9 \times 0^{-5} & E_{3} / E_{2}^{\mathrm{f}}=0.4 \\
G_{12} / E_{2}^{\mathrm{f}}=2.4 \times 0^{-3} & G_{13} / E_{2}^{\mathrm{f}}=7.9 \times 0^{-2} & G_{23} / E_{2}^{\mathrm{f}}=6.6 \times 0^{-2} \\
v_{12}=0.99 & v_{13}=v_{23}=3 \times 0^{-5} & \alpha_{1}=\alpha_{2}=\alpha_{3}=1.36 \alpha_{0}
\end{array}
$$

where $E_{2}^{\mathrm{f}}$ refers to $E_{2}$ of face sheets. Stability parameters of thermal buckling are calculated using the present higher order models and first-order model. The results are presented along with three-dimensional elasticity solutions in Table 3. Slight reading errors may be involved in the given values of the elasticity solution because they are taken from published graphs. Two parameters are varied, namely, the slenderness ratio of the sandwich, $a / h$, and the thickness ratio of the face sheets, $h_{f} / h$, where $h_{\mathrm{f}}$ is the thickness of each of the face sheets.

The results of the HOST9 model show good agreement with elasticity solution, whereas the results of the FOST and HOST11 models do not. The HOST9 model slightly overpredicts the critical temperature. The errors for sandwiches with $a / h$ ratios equal to 10 and above vary from 0 to $6 \%$ as the thickness ratios of face sheets increase from 0.025 to 0.15 . In the case of very thick sandwiches $(a / h=5)$ the maximum error is about $10 \%$. The results of FOST are accurate for sandwiches with very thin face sheets $\left(h_{\mathrm{f}} / h=0.025\right)$. However, the errors in the FOST results increase more rapidly as the thickness ratios of the face sheets increase. For sandwiches with $a / h=5$ and $h_{\mathrm{f}} / h=0.15$, the error is about $60 \%$. HOST 11 underestimates the critical temperature with significant margin. In the case of sandwiches with relatively thick core $\left(h_{\mathrm{f}} / h \leq 0.075\right)$, the errors are about 10 to $12 \%$ for all values of $a / h$ considered. It should be noted that as the depth of the core decreases, the errors in results decrease. For sandwiches with $h_{\mathrm{f}} / h=0.15$, the results are much closer to the elasticity solution when compared to those obtained using the HOST9 and FOST models. 
Table 3 Stability parameter for critical temperature, $\lambda_{\mathrm{T}}=\alpha_{0} T_{\mathrm{cr}}$, of symmetric sandwiches with composite cross-ply face sheets $(a / b=1)$

\begin{tabular}{lrllll}
\hline & & & & \\
$h_{\mathrm{f}} / h$ & $a / h$ & {$[14,16]$} & HOST9 & HOST11 & FOST \\
\hline 0.025 & 20.00 & 0.0929 & 0.0930 & 0.0854 & 0.0928 \\
& 10.00 & 0.3220 & 0.3231 & 0.2885 & 0.3211 \\
& 6.66 & 0.5900 & 0.6020 & 0.5238 & 0.5952 \\
& 5.00 & 0.8512 & 0.8696 & 0.7404 & 0.8556 \\
\hline 0.050 & 20.00 & 0.0855 & 0.0860 & 0.0785 & 0.0868 \\
& 10.00 & 0.2737 & 0.2764 & 0.2426 & 0.2843 \\
& 6.66 & 0.4592 & 0.4727 & 0.4040 & 0.4964 \\
& 5.00 & 0.6096 & 0.6330 & 0.5336 & 0.6758 \\
\hline 0.075 & 20.00 & 0.0791 & 0.0794 & 0.0725 & 0.0815 \\
& 10.00 & 0.2358 & 0.2397 & 0.2100 & 0.2592 \\
& 6.66 & 0.3697 & 0.3860 & 0.3308 & 0.4383 \\
& 5.00 & 0.4692 & 0.4932 & 0.4189 & 0.5813 \\
\hline 0.100 & 20.00 & 0.0726 & 0.0735 & 0.0673 & 0.0768 \\
& 10.00 & 0.2072 & 0.2113 & 0.1863 & 0.2402 \\
& 6.66 & 0.3123 & 0.3262 & 0.2826 & 0.3993 \\
& 5.00 & 0.3820 & 0.4047 & 0.3921 & 0.5224 \\
\hline 0.150 & 20.00 & 0.0623 & 0.0639 & 0.0593 & 0.0686 \\
& 10.00 & 0.1632 & 0.1732 & 0.1560 & 0.2119 \\
& 6.66 & 0.2347 & 0.2556 & 0.2279 & 0.3479 \\
& 5.00 & 0.2805 & 0.3087 & 0.2748 & 0.4504 \\
\hline
\end{tabular}

${ }^{a}$ Results shown in this column are obtained by interpolation of published graphs.

Example 3: Symmetric, square sandwich panels comprised of angle-ply composite face sheets and a honeycomb central core are considered here for thermal buckling analysis. Each of the face sheets has 10 layers of antisymmetric lamination $\left[(\theta /-\theta)_{5}\right]$. However, the face sheets are arranged in such a way to make the entire sandwich panel symmetric with respect to the middle plane. The material properties considered are the same as those used in the preceding example.

The HOST9 model which determines critical temperature more accurately and almost comparable to the three-dimensional elasticity solution, is used here. Parametric studies are conducted to study the effect of boundary conditions and variations in geometric and lamination parameters on critical temperature. Four types of boundary conditions such as SS1, SS2, C1, and C2 given in Table 1 are considered. The fiber orientation angle, $\theta$, is varied from 0 to 90 degrees; and the thickness ratio of face sheets, $h_{\mathrm{f}} / h$ (with total thickness $h$ kept fixed), is varied from 0.025 to 0.1 . Typical results are shown in Figures 2 to 5 .

Figures $(2 a, b)$ show the variation of the thermal stability parameter, $\lambda_{\mathrm{T}}$, with fiber orientation for a thin $(a / h=20)$ sandwich panel under SS1 and C1 (movable edges) boundary conditions, respectively, for different values of thickness ratio of 

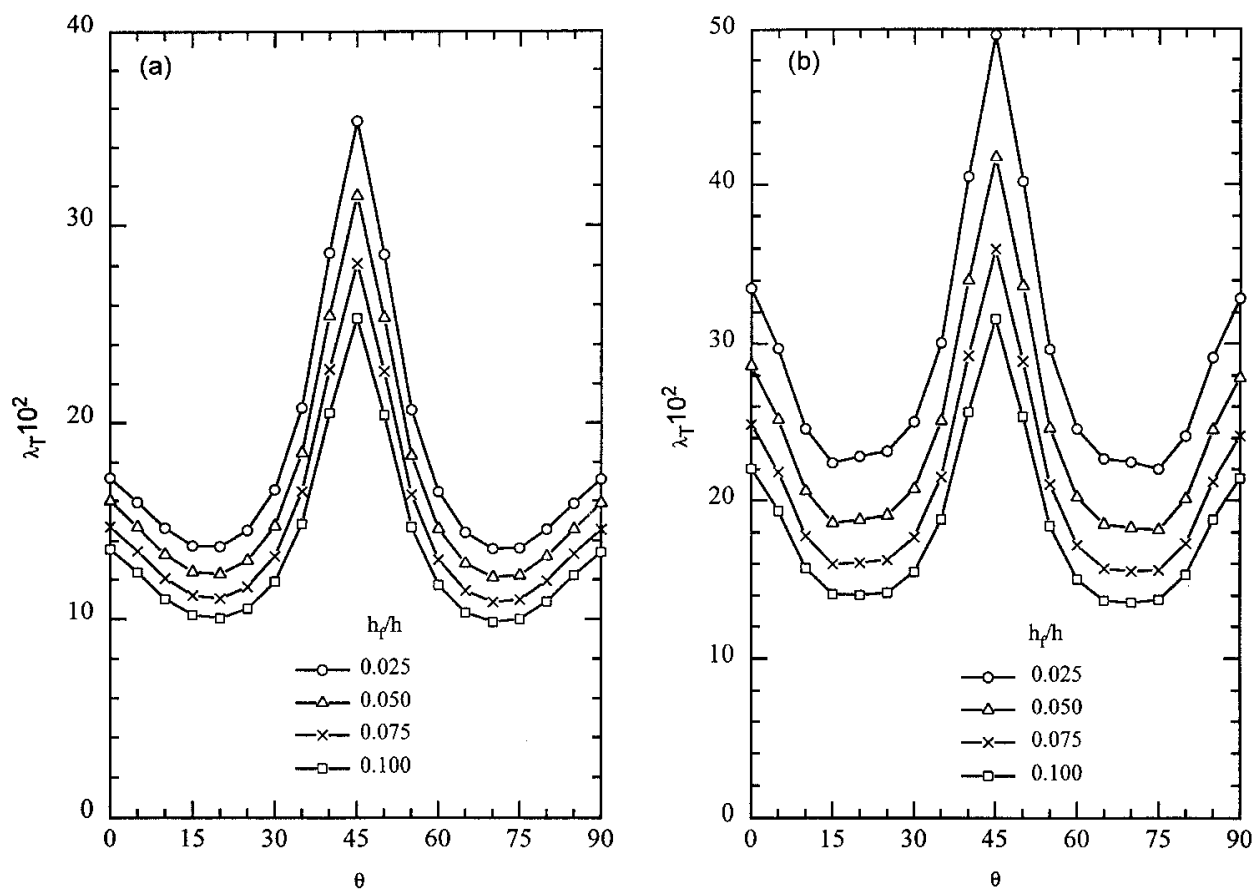

Figure 2. Effect of thickness ratio, $h_{\mathrm{f}} / h$, and fiber orientation angle, $\theta$, on the thermal stability parameter, $\lambda_{\mathrm{T}}=\alpha_{0} T_{\mathrm{cr}}$, of square sandwich panels $(a / h=20)$ : $(a) \mathrm{SS} 1,(b) \mathrm{C} 1$.

face sheets. Figures $3(a, b)$ show the variation of $\lambda_{\mathrm{T}}$ for the same sandwich panel under SS2 and C2 (immovable edges) boundary conditions, respectively. It can be observed that for given values of $h_{\mathrm{f}} / h$ and $\theta$, the critical temperature values of plates with movable edges (SS1 and $\mathrm{C} 1$ ) are greater than the corresponding values of plates with immovable edges (SS2 and C2). Note that the pattern of variation of critical temperature versus $\theta$ for the two kinds of boundary conditions, movable and immovable, are drastically different. It is well known that for angle-ply plates, the stiffness increases (thereby increasing critical temperature) as $\theta$ increases from 0 to 45 degrees and decreases thereafter as $\theta$ increases. This is clearly seen in the critical temperature values of plates with immovable edges (SS2 and C2). However, in case of plates with movable edges (SS1 and $\mathrm{C} 1$ ), the variation of critical temperature with $\theta$ is completely different from that of plates with immovable edges even though the stiffness quantities are the same for both the cases. Here, the critical temperature decreases as $\theta$ increases from 0 to approximately 20 degrees and thereafter increases as $\theta$ increases until $\theta=45$ degrees. The reason for this different nature of variation of $\lambda_{\mathrm{T}}$ between SS1 and SS2 or C1 and C2 boundary conditions is the variation of initial stress resultants $\left(N_{x}^{0}, N_{y}^{0}\right)$ with $\theta$. In case of plates with immovable edges, the stress resultants remain uniform throughout the plate. But for plates with movable edges, the stress resultants exhibit a nonuniform distribution. As $\theta$ increases from 0 to 20 degrees, the stress resultants 

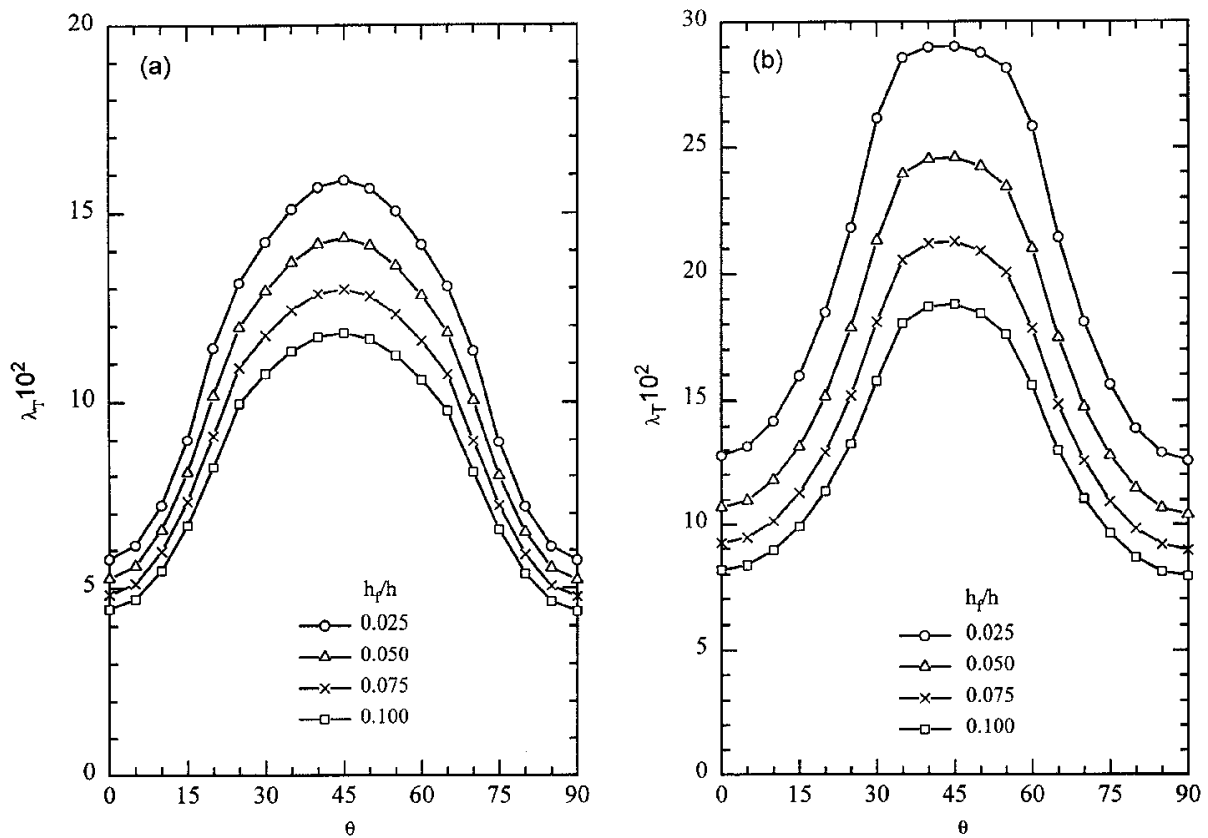

Figure 3. Effect of thickness ratio, $h_{\mathrm{f}} / h$, and fiber orientation angle, $\theta$, on the thermal stability parameter, $\lambda_{\mathrm{T}}=\alpha_{0} T_{\mathrm{cr}}$, of square sandwich panels $(a / h=20):(a) \mathrm{SS} 2,(b) \mathrm{C} 2$.
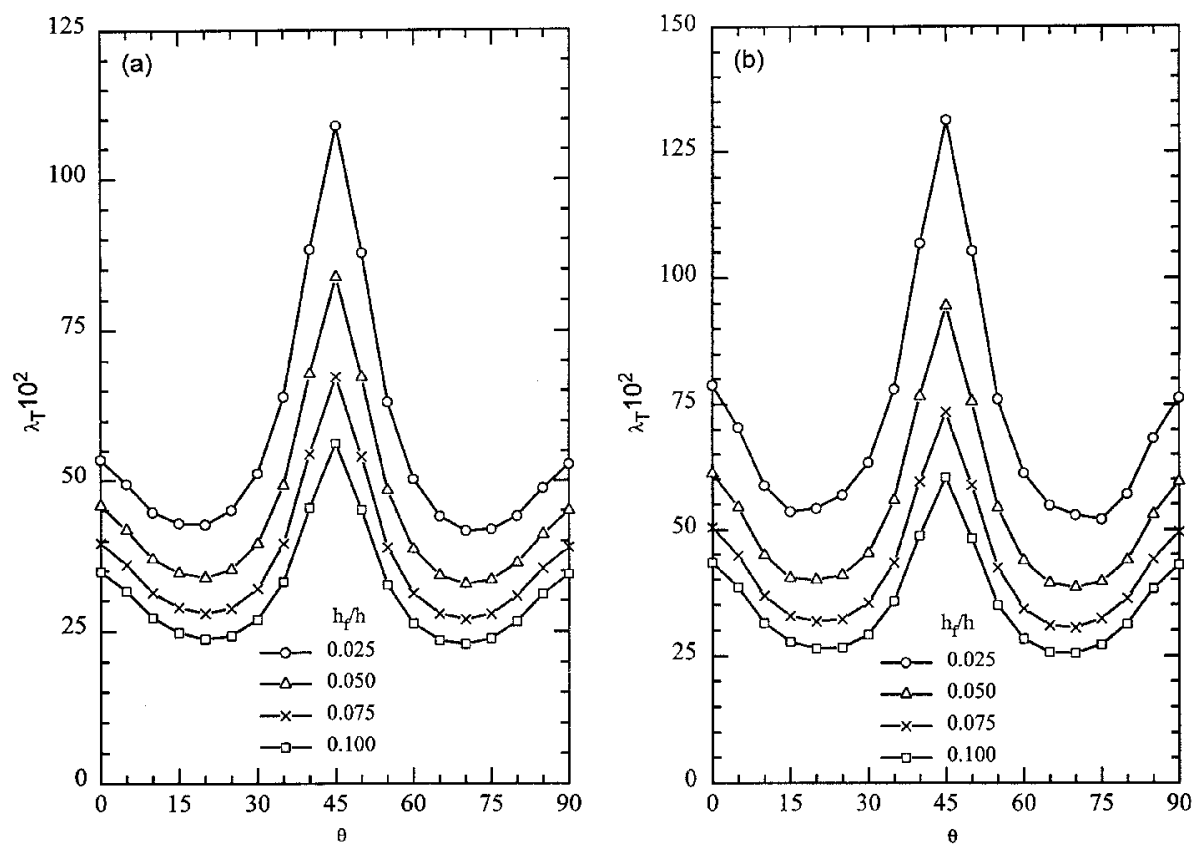

Figure 4. Effect of thickness ratio, $h_{\mathrm{f}} / h$, and fiber orientation angle, $\theta$, on the thermal stability parameter, $\lambda_{\mathrm{T}}=\alpha_{0} T_{\mathrm{cr}}$, of square sandwich panels $(a / h=10)$ : (a) SS1, (b) C1. 

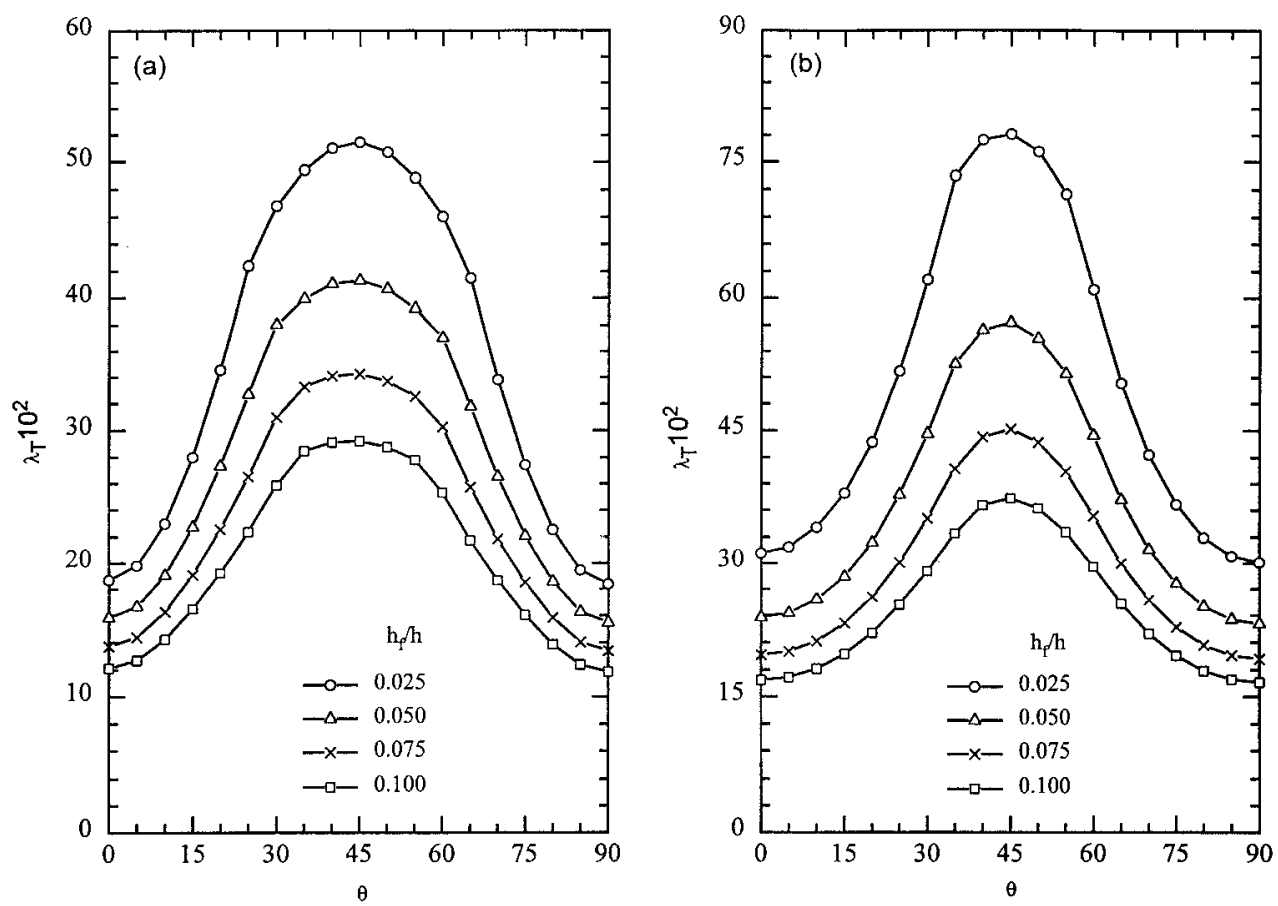

Figure 5. Effect of thickness ratio, $h_{\mathrm{f}} / h$, and fiber orientation angle, $\theta$, on the thermal stability parameter, $\lambda_{\mathrm{T}}=\alpha_{0} T_{\mathrm{cr}}$, of square sandwich panels $(a / h=10)$ : (a) SS2, (b) C2.

increase, and the increase is more pronounced than the increase in stiffness quantities. Due to this, the critical temperature decreases as $\theta$ increases from 0 to 20 degrees. Typical cases of distribution of stress resultants due to a unit change in temperature (with $\alpha_{0}=1$ ) for a plate under SS1 boundary conditions are shown in Figure 6. Figures $6(a-c)$ show the distribution of stress resultants along the center line parallel to the $x$-axis for $\theta=0,20$ and 45 degrees, respectively. From these figures it is understood that the stress resultants increase as $\theta$ increases from 0 to 20 degrees, reaching a maximum at $\theta=20$ degrees, and subsequently decrease as $\theta$ increases. Thus the nature of distribution of the stress resultants explains the cause for reduction in $\lambda_{\mathrm{T}}$ for plates with $\theta=20$ degrees fiber orientation under SS1 and $\mathrm{C} 1$ boundary conditions.

Figures 4 and 5 show the variation of $\lambda_{\mathrm{T}}$ with $\theta$ for thick $(a / h=10)$ sandwich plates under SS1, C1 and SS2, C2 boundary conditions, respectively. It should be noted that for thin and thick plates the variation in $\lambda_{\mathrm{T}}$ at around $\theta=45$ degree fiber orientation is sharp for SS1, C1 boundary conditions in comparison to a relatively smooth change for SS2, C2 boundary conditions. Another important observation is that for thick and thin panels, the critical temperature decreases as $h_{\mathrm{f}} / h$ increases. 


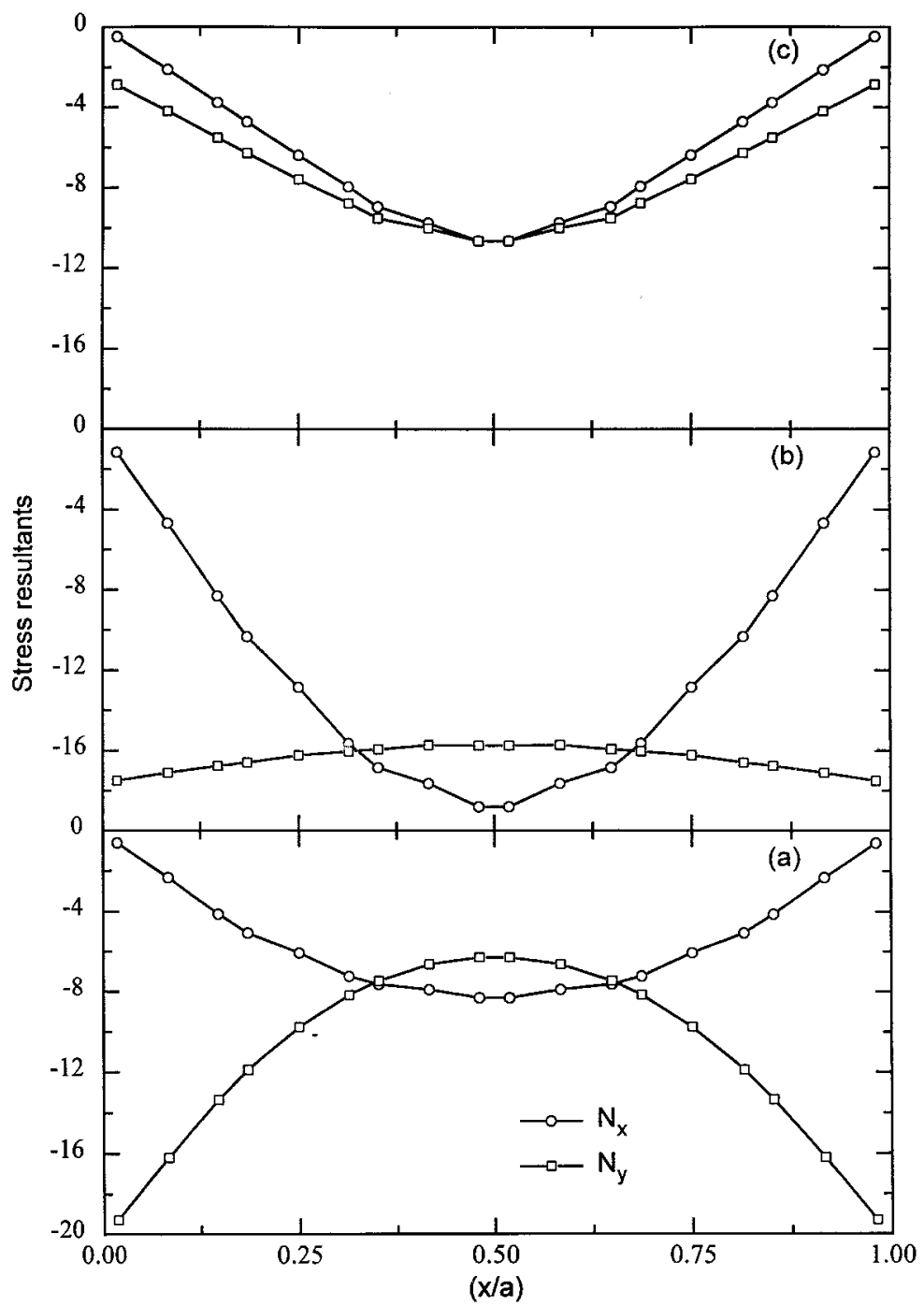

Figure 6. Variation of stress resultants at $y=b / 2$ with the $x$ coordinate in a sandwich panel $(a / h=20$, $h_{\mathrm{f}} / h=0.025$, BC: SS1): (a) $\theta=0^{\circ}$, (b) $\theta=20^{\circ}$, and (c) $\theta=45^{\circ}$ for $N_{x}=N_{x}^{0} b^{2} / E_{2} h^{3}$ and $N_{y}=$ $N_{y}^{0} b^{2} / E_{2} h^{3}$.

\section{CONCLUSIONS}

Two simple $C^{0}$ isoparametric finite element formulations based on higher order shear deformation theories are presented for the thermal buckling analysis of composite and sandwich laminates. The accuracy of the present models is evaluated for composite and sandwich laminates by comparison with the available three-dimensional elasticity solutions. The effects of various boundary conditions 
and geometric and lamination parameters on the critical temperature of sandwich panels are presented. The general conclusions based on the presented numerical results are summarized as follows.

- The present higher order theories have shown excellent agreement with the three-dimensional elasticity solution for both thin and thick composite laminates. However, in the case of sandwiches, HOST9 predicts more accurate results when compared to HOST11. Hence, transverse normal strain/deformation and thermal expansion in the thickness direction can be neglected in thermal buckling analyses.

- In the case of composite laminates, the results of FOST matches well those of present higher order models. For sandwiches, FOST overestimates the critical temperature and the errors are significant when compared to those of HOST9.

- In symmetric sandwich panels with angle-ply composite face sheets subjected to uniform temperature distribution, the internal thermal stress field is nonuniform for SS1, C1 boundary conditions and uniform for SS2, C2 boundary conditions.

- The thermal buckling strength of symmetric, square sandwiches consisting of angle-ply face sheets is maximum for $\theta=45$ degrees fiber orientation. For thin and thick sandwich panels, an increase in face sheet thickness results in a decrease in critical temperature.

\section{REFERENCES}

1. T. R. Tauchert, Thermally Induced Flexure, Buckling, and Vibration of Plates, Appl. Mech. Revs., vol. 44, pp. 347-360, 1991.

2. A. K. Noor and W. S. Burton, Computational Models for High-Temperature Multilayered Composite Plates and Shells, Appl. Mech. Revs., vol. 45, pp. 419-446, 1992.

3. E. A. Thornton, Thermal Buckling of Plates and Shells, Appl. Mech. Revs., vol. 46, pp. 485-506, 1993.

4. T. R. Tauchert, Thermal Buckling of Thick Antisymmetric Angle-Ply Laminates, J. Thermal Stresses, vol. 10, pp. 113-124, 1987.

5. I.-H. Y ang and J.-A. Shieh, Generic Thermal Buckling of Initially Stressed Antisymmetric Cross-Ply Thick Laminates, Int. J. Solids Struct., vol. 24, pp. 1059-1070, 1988.

6. W. J. Chen, P. D. Lin, and L. W. Chen, Thermal Buckling Behaviour of Thick Composite Laminated Plates under Nonuniform Temperature Distribution, Computers and Struct., vol. 41, pp. 637-645, 1991.

7. A. K. Noor and W. S. Burton, Predictor-Corrector Procedures for Thermal Buckling Analysis of Multilayered Composite Plates, Computers and Struct., vol. 40, pp. 1071-1084, 1991.

8. M. R. Prabhu and R. C. Dhanaraj, Thermal Buckling of Laminated Composite Plates, Computers and Struct., vol. 53, pp. 1193-1204, 1994.

9. J.-S. Chang, FEM Analysis of Buckling and Thermal Buckling of Antisymmetric Angle-Ply Laminates according to Transverse Shear and Normal Deformable High Order Displacement Theory, Computers and Struct., vol. 37, pp. 925-946, 1990.

10. J.-S. Chang and S.-Y. Leu, Thermal Buckling Analysis of Antisymmetric Angle-Ply Laminates in a Uniform-Temperature Field, Compos. Sci. Tech., vol. 41, pp. 109-128, 1991.

11. J. N. Reddy, A Simple Higher-Order Theory for Laminated Composite Plates, ASME J. Appl. Mech., vol. 51, pp. 745-752, 1984.

12. K. Rohwer, Discussion of Chang and Leu, Compos. Sci. Tech., vol. 45, pp. 181-182, 1992. 
13. X. Shu and L. X. Sun, Thermomechanical Buckling of Laminated Composite Plates with HigherOrder Transverse Shear Deformation, Computers and Struct., vol. 53, pp. 1-8, 1994.

14. A. K. Noor, J. M. Peters, and W. S. Burton, Three-Dimensional Solutions for Initially Stressed Structural Sandwiches, ASCE J. Engrg. Mech., vol. 120, pp. 284-303, 1994.

15. W. L. Ko and R. H. Jackson, Compressive and Shear Buckling Analysis of Metal Matrix Composite Sandwich Panels under Different Thermal Environments, Composite Struct., vol. 25, pp. 227-239, 1993.

16. A. K. Noor, W. S. Burton, and C. W. Bert, Computational Models for Sandwich Panels and Shells, Appl. Mech. Revs., vol. 49, pp. 155-199, 1996.

17. T. Kant, Numerical Analysis of Thick Plates, Comput. Meth. Appl. Mech. Engrg., vol. 31, pp. 1-18, 1982.

18. T. Kant, D. R. J. Owen, and O. C. Zienkiewicz, A Refined Higher-Order $C^{\circ}$ Plate Bending Element, Computers and Struct., vol. 15, pp. 177-183, 1982.

19. T. Kant and B. N. Pandya, A Simple Finite Element Formulation of a Higher-Order Theory for Unsymmetrically Laminated Composite Plates, Composite Struct., vol. 9, pp. 215-246, 1988.

20. T. Kant and Mallikarjuna, A Higher-Order Theory for Free Vibration of Unsymmetrically Laminated Composite and Sandwich Plates-Finite Element Evaluations, Computers and Struct., vol. 32, pp. 1125-1132, 1989a.

21. T. Kant and Mallikarjuna, Vibration of Unsymmetrically Laminated Plates Analyzed by Using a Higher-Order Theory with a $C^{\circ}$ Finite Element Formulation, J. Sound and Vibration, vol. 134, pp. $1-16,1989 \mathrm{~b}$.

22. T. Kant and R. K. Khare, Finite Element Thermal Stress Analysis of Composite Laminates Using a Higher-Order Theory, J. Thermal Stresses, vol. 17, pp. 229-255, 1994.

23. J. R. Vinson and R. L. Sierakowski, The Behaviour of Structures Composed of Composite Materials, Martinus Nijhoff, Dordrecht, 1986.

24. O. C. Zienkiewicz and R. L. Taylor, The Finite Element Method, vol. 1, 4th ed., McGraw-Hill, Singapore, 1989.

25. A. K. Noor and W. S. Burton, Three-Dimensional Solutions for Thermal Buckling of Multilayered Anisotropic Plates, ASCE J. Engrg. Mech., vol. 118, pp. 683-701, 1992.

\section{APPENDIX}

The components of the strain vector, $\overline{\boldsymbol{\epsilon}}$, in Eqs. (10) and (13) are

$$
\bar{\epsilon}=\left\{\begin{array}{lll}
\boldsymbol{\epsilon}_{0}^{\mathbf{T}} & \chi^{\mathrm{T}} \quad \boldsymbol{\psi}^{\mathrm{T}}
\end{array}\right\}^{\mathrm{T}}
$$

where

$$
\begin{aligned}
\boldsymbol{\epsilon}_{0} & =\left\{\varepsilon_{x}^{0}, \varepsilon_{y}^{0}, \varepsilon_{x y}^{0}, \varepsilon_{x}^{*}, \varepsilon_{y}^{*}, \varepsilon_{x y}^{*}, \varepsilon_{x}^{* *}, \varepsilon_{y}^{* *}, \varepsilon_{x y}^{* *}, \varepsilon_{x}^{* * *}, \varepsilon_{y}^{* * *}, \varepsilon_{x y}^{* * *}\right\}^{\mathrm{T}} \\
\chi & =\left\{\chi_{x}^{0}, \chi_{y}^{0}, \chi_{x y}^{0}, \chi_{x}^{*}, \chi_{y}^{*}, \chi_{x y}^{*}, \chi_{x}^{* *}, \chi_{y}^{* *}, \chi_{x y}^{* *}\right\}^{\mathrm{T}} \\
\Psi & =\left\{\phi_{x}^{0}, \phi_{y}^{0}, \psi_{x}^{0}, \psi_{y}^{0}, \phi_{x}^{*}, \phi_{y}^{*}, \psi_{x}^{*}, \psi_{y}^{*}, \phi_{x}^{* *}, \phi_{y}^{* *}, \psi_{x}^{* *}, \psi_{y}^{* *}\right\}^{\mathrm{T}}
\end{aligned}
$$

The components of the stress resultant vector, $\bar{\sigma}$, in Eq. (13) are

$$
\bar{\sigma}=\left\{\begin{array}{lll}
\mathbf{N}^{\mathrm{T}} & \mathbf{M}^{\mathrm{T}} & \mathbf{Q}^{\mathrm{T}}
\end{array}\right\}^{\mathrm{T}}
$$


where

$$
\begin{aligned}
& \mathbf{N}=\left\{N_{x}^{0}, N_{y}^{0}, N_{x y}^{0}, N_{x}^{*}, N_{y}^{*}, N_{x y}^{*}, N_{x}^{* *}, N_{y}^{* *}, N_{x y}^{* *}, N_{x}^{* * *}, N_{y}^{* * *}, N_{x y}^{* * *}\right\}^{\mathrm{T}} \\
& \mathbf{M}=\left\{M_{x}^{0}, M_{y}^{0}, M_{x y}^{0}, M_{x}^{*}, M_{y}^{*}, M_{x y}^{*}, M_{x}^{* *}, M_{y}^{* *}, M_{x y}^{* *}\right\}^{\mathrm{T}} \\
& \mathbf{Q}=\left\{Q_{x}^{0}, Q_{y}^{0}, S_{x}^{0}, S_{y}^{0}, Q_{x}^{*}, Q_{y}^{*}, S_{x}^{*}, S_{y}^{*}, Q_{x}^{* *}, Q_{y}^{* *}, S_{x}^{* *}, S_{y}^{* *}\right\}^{\mathrm{T}}
\end{aligned}
$$

The components of thermal stress resultant vector, $\bar{\sigma}_{t}$ in Eq. (13) are

$$
\bar{\sigma}_{\mathrm{t}}=\left\{\begin{array}{lll}
\mathbf{N}_{\mathrm{t}}^{\mathrm{T}} & \mathbf{M}_{\mathrm{t}}^{\mathrm{T}} & \mathbf{O}_{\mathrm{t}}^{\mathrm{T}}
\end{array}\right\}^{\mathrm{T}}
$$

where

$$
\begin{aligned}
& \mathbf{N}_{\mathrm{t}}=\left\{N_{\mathrm{t} x}^{0}, N_{\mathrm{t} y}^{0}, N_{\mathrm{t} x y}^{0}, N_{\mathrm{t} x}^{*}, N_{\mathrm{t} y}^{*}, N_{\mathrm{t} x y}^{*}, N_{\mathrm{t} x}^{* *}, N_{\mathrm{t} y}^{* *}, N_{\mathrm{t} x y}^{* *}, N_{\mathrm{t} x}^{* * *}, N_{\mathrm{t} y}^{* * *}, N_{\mathrm{t} x y}^{* * *}\right\}^{\mathrm{T}} \\
& \mathbf{M}_{\mathrm{t}}=\left\{M_{\mathrm{t} x}^{0}, M_{\mathrm{t} y}^{0}, M_{\mathrm{t} x y}^{0}, M_{\mathrm{t} x}^{*}, M_{\mathrm{t} y}^{*}, M_{\mathrm{t} x y}^{*}, M_{\mathrm{t} x}^{* *}, M_{\mathrm{t} y}^{* *}, M_{\mathrm{t} x y}^{* *}\right\}^{\mathrm{T}} \\
& \mathbf{O}_{\mathrm{t}}=\{0,0,0,0,0,0,0,0,0,0,0,0\}^{\mathrm{T}}
\end{aligned}
$$

in which

$$
\mathbf{N}_{\mathrm{t}}=\sum_{L=1}^{N L}\left[\begin{array}{c}
Q_{i j} H_{1} \\
Q_{i j} H_{3} \\
Q_{i j} H_{5} \\
Q_{i j} H_{7}
\end{array}\right]\left\{\begin{array}{c}
\alpha_{x} \\
\alpha_{y} \\
\alpha_{x y}
\end{array}\right\} \Delta T \text { and } \mathbf{M}_{\mathrm{t}}=\sum_{L=1}^{N L}\left[\begin{array}{c}
Q_{i j} H_{2} \\
Q_{i j} H_{4} \\
Q_{i j} H_{6}
\end{array}\right]\left\{\begin{array}{c}
\alpha_{x} \\
\alpha_{y} \\
\alpha_{x y}
\end{array}\right\} T
$$

The rigidity matrices in Eq. (18) are

$$
\begin{aligned}
\mathbf{D}_{m}=\sum_{L=1}^{N L}\left[\begin{array}{llll}
Q_{i j} H_{1} & Q_{i j} H_{3} & Q_{i j} H_{5} & Q_{i j} H_{7} \\
& Q_{i j} H_{5} & Q_{i j} H_{7} & Q_{i j} H_{9} \\
& & Q_{i j} H_{9} & Q_{i j} H_{11} \\
\text { Sym } & & & Q_{i j} H_{13}
\end{array}\right] \\
\mathbf{D}_{c}=\sum_{L=1}^{N L}\left[\begin{array}{lll}
Q_{i j} H_{2} & Q_{i j} H_{4} & Q_{i j} H_{6} \\
Q_{i j} H_{4} & Q_{i j} H_{6} & Q_{i j} H_{8} \\
Q_{i j} H_{6} & Q_{i j} H_{8} & Q_{i j} H_{10} \\
Q_{i j} H_{8} & Q_{i j} H_{10} & Q_{i j} H_{12}
\end{array}\right]
\end{aligned}
$$




$$
\begin{aligned}
& \mathbf{D}_{b}=\sum_{L=1}^{N L}\left[\begin{array}{ccc}
Q_{i j} H_{3} & Q_{i j} H_{5} & Q_{i j} H_{7} \\
& Q_{i j} H_{7} & Q_{i j} H_{9} \\
\text { Sym } & & Q_{i j} H_{11}
\end{array}\right] \\
& \mathbf{D}_{s}=\sum_{L=1}^{N L}\left[\begin{array}{cccccc}
Q_{m l} H_{1} & Q_{m l} H_{2} & Q_{m l} H_{3} & Q_{m l} H_{4} & Q_{m l} H_{5} & Q_{m l} H_{6} \\
& Q_{m l} H_{3} & Q_{m l} H_{4} & Q_{m l} H_{5} & Q_{m l} H_{6} & Q_{m l} H_{7} \\
& & Q_{m l} H_{5} & Q_{m l} H_{6} & Q_{m l} H_{7} & Q_{m l} H_{8} \\
& & & Q_{m l} H_{7} & Q_{m l} H_{8} & Q_{m l} H_{9} \\
& & & & Q_{m l} H_{9} & Q_{m l} H_{10} \\
& & & & & Q_{m l} H_{11}
\end{array}\right] \text { (Cont.) }
\end{aligned}
$$

where

$$
i, j=1,2,3 \quad l, m=4,5 \quad H_{i}=\frac{\left(z_{L+1}^{i}-z_{L}^{i}\right)}{i}
$$

